\title{
Ignition of a Thermonuclear Detonation Wave in the Focus of Two Magnetically Insulated Transmission Lines
}

\author{
F. Winterberg \\ Department of Physics, University of Nevada, Reno, Nevada, 89551 U. S. A. \\ Reprint requests to Prof. F. W.; Fax: (775)784-1398, eMail: winterbe@physics.unr.edu
}

Z. Naturforsch. 58a, 197 - 200 (2003); received February 5, 2003

For the ignition of a thermonuclear detonation wave assisted by a strong magnetic field, it is proposed to use two concentrically nested magnetically insulated transmission lines, the inner one transmitting a high- voltage lower-current-, and the outer one a high-current lower-voltage- electromagnetic pulse drawn from two Marx generators. The concept has the potential of large thermonuclear gains with an input energy conceivably as small as $10^{5} \mathrm{~J}$.

Key words: Nuclear Fusion; Fast Ignition.

\section{Introduction}

The ultimate goal of controlled nuclear fusion by inertial confinement is a low- yield, high-gain thermonuclear microexplosion assembly, with the low yield required for the confinement of the microexplosion in a cavity of manageable size, and the high gain demanded for an efficient release of thermonuclear energy.

In thermonuclear microexplosions a high gain can be achieved with a thermonuclear detonation wave ignited from a "hot spot." In the absence of a magnetic field this requires the precompression of the fusion fuel to $\sim 10^{3}$ times solid density, with the hot spot created in the center of the precompressed fuel by a convergent shock wave [1] or, as in the fast ignitor concept [2], by a pulsed ultraintense laser beam. In the presence of a strong magnetic field the range of the charged fusion products is replaced by their Larmor radius, which can be much smaller, making possible a propagating thermonuclear burn in small assemblies even at less than solid densities. In general though, much more energy is needed here for ignition, in particular for magnetized target fusion, except for configurations where the magnetized fusion target is used to trigger a much larger thermonuclear microexplosion [3]. A different idea is to ignite a thermonuclear detonation wave in a lowtemperature, high-density shear flow stabilized fast zpinch, with the hot spot for ignition created by a pulsed laser beam [4]. But even there the overall gain is limited mainly because this concept requires the magnetic plasma confinement along the entire pinch discharge channel.
About 35 years ago [5] I had shown that the ignition of a thermonuclear microexplosion should be possible by the bombardment of a small solid DT target with an intense $\left(10^{8} \mathrm{~A}, 10^{7} \mathrm{~V}\right)$ relativistic electron beam drawn from a large Marx generator, and it was found that breakeven would require a beam energy of about $10 \mathrm{MJ}$, to be delivered to a less than $\mathrm{cm}$-size target in about $10^{-8}$ seconds. ${ }^{1}$ More specifically, it was proposed to place the target inside a hollowed-out metallic tamp, with the beam energy dissipated in the target by the electrostatic two-stream instability.

It is the purpose of this communication to show that with two Marx generators and two concentrically nested magnetically insulated transmission lines, the inner one transmitting a high voltage lower current-, and the outer one a high current lower voltage-, pulse, a thermonuclear detonation wave with a high gain can be ignited in the common focus of the two transmission lines, and with an input energy as small as $10^{5} \mathrm{~J}$.

\section{The Proposed Novel Concept}

As shown in Fig. 1 the inner transmission line (left) ends in a cone with the tip of the cone serving as a cathode for a field emitted intense relativistic electron beam. The return current conductor of the inner transmission line is at its smallest diameter connected to a cone of solid DT, serving as the anode of the inner transmission line. An intense relativistic electron beam

\footnotetext{
${ }^{1}$ By order of magnitude these numbers still hold and are about the same as for the planned X1 machine.
} 
198

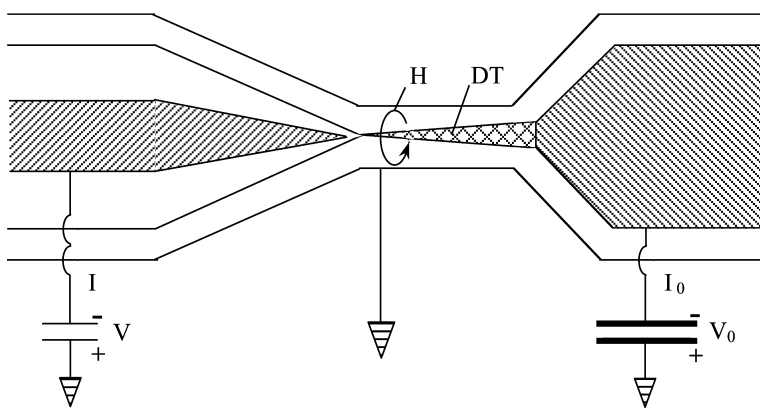

Fig. 1. The two nested magnetically insulated transmission lines with the DT target ignited in the focus of both lines. In our example we have chosen for the inner line a voltage of $V=10^{7} \mathrm{~V}$ and current $I=10^{5} \mathrm{~A}$; and for the outer line $V_{0}=10^{6} \mathrm{~V}$ and $I_{0}=10^{7} \mathrm{~A}$.

emitted from the cathode tip of the inner line is by selfmagnetic forces focused onto the DT cone, heating the tip of the cone to thermonuclear temperatures by the electrostatic two-stream instability. At the same time, a large electric current is discharged through the outer transmission line, with the current passing over the DT cone and the outer side of the conical segment of the inner transmission line. This current must be large enough to generate at the focus of both transmission lines a magnetic pressure which can balance the pressure of the DT plasma at thermonuclear temperatures. In addition to heating the DT plasma, the energy from the relativistic electron beam emitted from the end of the inner transmission line must compensate the axial expansion losses of the hot plasma blown off to the left from the tip of the DT cone. If both conditions are met, a shock wave moves to the right into the DT cone, and if the charged fusion reaction $\alpha$-particles are confined within the cone by the magnetic field of the current flowing over the cone, the shock wave goes over into a thermonuclear detonation wave supersonically moving down the cone. Except for the small region near the vertex of the DT cone, no magnetic plasma confinement is required, with the magnetic field otherwise serving only to entrap the charged fusion reaction $\alpha$ particles in the DT cone. It is for that reason that large fusion gains can here be reached.

\section{Plasma Heating by the Intense Relativistic Electron Beam}

The current density of the field emitted electrons is [6]
F. Winterberg · Ignition of a Thermonuclear Detonation Wave

$$
\begin{aligned}
j= & 1.55 \cdot 10^{-8}\left(E^{2} / W\right) \\
& \cdot \exp \left(-6.9 \cdot 10^{7} W^{3 / 2} / E\right) \mathrm{A} / \mathrm{cm}^{2},
\end{aligned}
$$

where $E$ is the electric field at the cathode in $\mathrm{V} / \mathrm{cm}$ and $W$ the work function in eV. For a semispherical cathode tip of radius $r$ one has $E \cong V / r$, where $V$ is the electric potential of the cathode. If $W=4.4 \mathrm{eV}$ as for tungsten, one has for the total current emitted from a semispherical surface area $2 \pi r^{2}$

$$
I=2.2 \cdot 10^{-6} V^{2} \exp \left(-6.4 \cdot 10^{8} r / V\right) \mathrm{A} .
$$

As an example we take $V=10^{7} \mathrm{~V}, r=0.1 \mathrm{~cm}$ and find $I=3.5 \cdot 10^{5} \mathrm{~A}$. With a beam radius of $0.1 \mathrm{~cm}$, the current density is $j \cong 10^{7} \mathrm{~A} / \mathrm{cm}^{2}$. By the selfmagnetic field and the repulsive image currents in the convergent conical return current conductor, the beam can be focused down to an even smaller diameter, with the axial electric field from the cathode tip to the anode preventing the beam from being reflected back to the cathode. Assuming that the beam can in this way be focused down to a radius of $r_{0}=10^{-2} \mathrm{~cm}$, the current density would rise to $\sim 10^{9} \mathrm{~A} / \mathrm{cm}^{2}$, with an electron number density in the beam $n_{\mathrm{b}} \cong 0.7 \cdot 10^{17} \mathrm{~cm}^{-3}$.

The e-fold beam stopping length by the collective electrostatic two-stream instability is [7]

$$
\lambda_{\mathrm{c}}=\frac{1.4 c \gamma}{\omega_{\mathrm{p}} \varepsilon^{1 / 3}}, \varepsilon=\frac{n_{\mathrm{b}}}{n_{0}}, \gamma=\left(1-v^{2} / c^{2}\right)^{-1 / 2}
$$

where for solid DT with $n_{0}=5 \cdot 10^{22} \mathrm{~cm}^{-3}$ one has $\omega_{\mathrm{p}}=\sqrt{4 \pi n_{0} e^{2} / m_{0}}=1.3 \cdot 10^{16} \mathrm{~s}^{-1}$, with the relativistic factor $\gamma$ taking into account the longitudinal electron mass $m=\gamma^{3} m_{0}$. For $10 \mathrm{MeV}$ electrons with $\gamma \approx 20$ one finds that $\lambda_{\mathrm{c}}=6 \cdot 10^{-3} \mathrm{~cm}$. This means that just the tip of the DT cone is heated.

A shock, propagating in the presence of a strong magnetic field in a direction perpendicular to this field, becomes a collisionless shock with a shock- thickness of the order of the plasma ion gyroradius [8]. For a DT plasma at a temperature of $10 \mathrm{keV}$ and a magnetic field of $10^{8} \mathrm{G}$ (the latter produced by the large current passing through the outer transmission line), the gyroradius is of the order $10^{-4} \mathrm{~cm}$. This collisionless shock will aid the two-stream instability in the rapid dissipation of the kinetic electron beam energy.

\section{Ignition}

The conditions for ignition are: 
1. Under steady state the power flux density of the incoming electron beam

$$
\phi_{\text {in }}=I V / \pi r_{0}^{2}
$$

must balance the power flux density of the ablated DT:

$$
\phi_{\text {out }}=2 n_{0} \frac{M v^{2}}{2} \frac{v}{6}=\frac{1}{6} \rho v^{3},
$$

where $v$ is the nondirectional ablation velocity, with the fraction 1/6 going in one direction. $M$ is the mass of the DT nuclei and $\rho=0.21 \mathrm{~g} / \mathrm{cm}^{3}$ the density of solid DT. For $V=10^{7} \mathrm{~V}, I=10^{5} \mathrm{~A}, r_{0}=10^{-2} \mathrm{~cm}$, one finds that $\phi_{\text {in }} \cong 3 \cdot 10^{22} \mathrm{erg} / \mathrm{cm}^{2} \mathrm{~s}$. Equating $\phi_{\text {out }}$ with $\phi_{\text {in }}$ one finds that $v \cong 10^{8} \mathrm{~cm} / \mathrm{s}$.

2. For $v \cong 10^{8} \mathrm{~cm} / \mathrm{s}$ the kinetic temperature of the DT ions is

$$
T=M v^{2} / 4 k \cong 10^{80} \mathrm{~K},
$$

about equal the ignition temperature of the DT thermonuclear reaction.

3. Equating the plasma pressure with the magnetic pressure $H_{0}^{2} / 8 \pi$ at $r=r_{0}$, generated by the current $I_{0}$ passing through the outer transmission line, one finds from

$$
2 n_{0} k T=H_{0}^{2} / 8 \pi
$$

that

$$
H_{0}=1,9 \cdot 10^{8} \mathrm{G} \text { and } I_{0}=5 r_{0} H_{0} \cong 10^{7} \mathrm{~A} .
$$

4. To confine the charged DT fusion reaction $\alpha$-particles within the DT cone requires that [9]

$$
r \ll r_{\mathrm{L}}=\frac{a}{H}, a=2.7 \cdot 10^{5}[\mathrm{Gcm}],
$$

where $r_{\mathrm{L}}$ is the Larmor radius of the charged fusion $\alpha$ particles and $r$ the radius of the DT cone at any position along the cone. With $H=0.2 I_{0} / r$, this requires that $I_{0} \gg 5 a=1.35 \cdot 10^{6} \mathrm{~A}$, well satisfied for $I_{0}=10^{7} \mathrm{~A}$.

5. Approximating the magnetically confined tip of the DT cone by a cylinder of radius $r_{0}$ and height $2 r_{\mathrm{L}}$, the latter equalling the effective range of the fusion $\alpha$ particles, the condition for ignition is [9]

$$
E_{\text {ign }}>3 n_{0} k T \pi r_{0}^{2} 2 r_{\mathrm{L}}
$$

With $3 n_{0} k T=(3 / 2)\left(H_{0}^{2} / 8 \pi\right), H_{0}=0.2 I_{0} / r_{0}$ and $r_{\mathrm{L}}=$ $a / H_{0}$ one has

$$
E_{\mathrm{ign}}>(3 / 40) a r_{0} I_{0} \cong 2 \cdot 10^{9} \mathrm{erg}=200 \mathrm{~J} .
$$

6. To overcome the bremsstrahlungs losses the ignition energy has to be supplied by the relativistic electron beam in less than $\sim 10^{-8} \mathrm{~s}$.

The e-fold stopping length of the beam by the two stream instability, equal to $6 \cdot 10^{-3} \mathrm{~cm}$, is about twice as large as $2 r_{\mathrm{L}} \cong 3 \cdot 10^{-3} \mathrm{~cm}$, requiring that $E_{\text {ign }}>$ $400 \mathrm{~J}$. Existing beam technology permits to generate a $10^{7} \mathrm{~V}, 10^{5} \mathrm{~A}, 10^{12} \mathrm{~W}$ beam lasting $10^{-9} \mathrm{~s}$, and delivering $\sim 1 \mathrm{~kJ}$, sufficient for ignition, with the beam current below the Alfvén limit.

\section{Thermonuclear Yield and Gain}

A growing thermonuclear detonation wave in the DT cone without magnetic plasma confinement requires 1) that the rate at which the energy is released into charged fusion products is larger than the bremsstrahlungs-loss rate, and 2) that the charged fusion products dissipate their kinetic energy into the cone. The first condition is valid for the DT thermonuclear reaction, and the second condition is satisfied by the $10^{7}$ A current flowing along the cone.

If the cone has the height $h$, and the radius at its base is $R$, then there are $\left(\pi R^{2} h / 3\right)(n / 2)$ pairs of DT nuclei in the cone, releasing an energy equal to

$$
E_{\text {out }}=\left(\pi R^{2} h / 3\right)(n / 2) \varepsilon_{\mathrm{f}},
$$

where $\varepsilon_{\mathrm{f}}=17.4 \mathrm{MeV} \cong 2.8 \cdot 10^{-5} \mathrm{erg}$.

As an example we take a cone of height $h=1 \mathrm{~cm}$ and base radius $R=0.1 \mathrm{~cm}$. We find that $E_{\text {out }} \cong 1$ GJ. The input energy is essentially determined by the energy of the high current pulse, with the ignition energy of the high voltage pulse small in comparison. For $I_{0}=10^{7} \mathrm{~A}$ at a voltage $V_{0}=10^{6} \mathrm{~V}$ with a power of $10^{13} \mathrm{~W}$ lasting $10^{-8} \mathrm{~s}$, the input energy is $E_{\text {in }}=10^{5} \mathrm{~J}$, and the gain $E_{\text {out }} / E_{\text {in }}=10^{4}$. Smaller gains with a smaller yield are then, of course, also possible.

\section{The Inner and Outer Magnetically Insulated Transmission Line}

The impedance of a coaxial transmission line is

$$
Z=60 \log (b / a)[\Omega]
$$

where $a$ is the radius of the inner, and $b$ the radius of the outer conductor. In a magnetically insulated transmission line, the line impedance is matched to the current pulse passing through the line. For the inner transmission line we have $I=10^{5} \mathrm{~A}, V=10^{7} \mathrm{~V}$, requiring that 
$Z=100 \Omega$. Therefore, if $a=0.5 \mathrm{~cm}$, then $b=2.1 \mathrm{~cm}$, and if the ratio $b / a$ is kept constant in the tapered conical section, the impedance of the inner line will be $100 \Omega$.

For the outer transmission line $I=10^{7} \mathrm{~A}, V=10^{6} \mathrm{~V}$, and therefore $Z=0.1 \Omega$. Such a small impedance is not possible with a single line, but is possible with several lines in parallel.

\section{Stability}

Stability can here only be a problem near the focus of the two centered magnetically insulated transmission lines, where the conical section of the transmission lines and the DT cone meet, because only there the plasma pressure must be balanced by the magnetic pressure. Shear flow stabilization by supersonic jets generated through magnetic implosion [10] in the vicinity of the focus should solve this remaining problem.

\section{Discussion}

It may be difficult to focus an intense relativistic electron beam down to a diameter as small as $\sim$ $10^{-2} \mathrm{~cm}$. With an ignition energy of $\sim 200 \mathrm{~J}$ the ignition could still be done with a laser beam, but also with a less focused electron beam by making some

[1] J. Nuckolls, L. Wood, and G. Zimmerman, Nature London 239, 139 (1972).

[2] M. Tabak et al., Physics of Plasmas 1, 1626 (1994).

[3] F. Winterberg, Z. Naturforsch. 39a, 325 (1984).

[4] F. Winterberg, Z. Naturforsch. 53a, 933 (1998).

[5] F. Winterberg, Phys. Rev. 174, 212 (1968).

[6] W. Finkelnburg, Structure of Matter, Academic Press, New York 1964. simple modifications. A ten times larger beam diameter, which seems technically feasible, would according to (10) increase the ignition energy ten-fold to $2 \mathrm{~kJ}$. The density of the DT at the location of ignition would have to be reduced $\sim 100$ fold, from $5 \cdot 10^{22} \mathrm{~cm}^{-3}$ (solid state) to $\sim 5 \cdot 10^{20} \mathrm{~cm}^{-3}$, otherwise the magnetic pressure can not balance the plasma pressure. At the $\sim 100$ fold reduced plasma density, the bremsstrahlungs - loss time would be increased $\sim 100$ fold from $\sim 10^{-8} \mathrm{~s}$ to $\sim 10^{-6} \mathrm{~s}$. Within $10^{-8} \mathrm{~s}$, which is shorter than the bremsstrahlungs - loss time, a $10^{12}$ $\mathrm{W}$ beam would deliver $10 \mathrm{~kJ}$, more than what is required for ignition. With the DT density inversely proportional to $r_{0}^{2}$, the ratio $\phi_{\text {out }} / \phi_{\text {in }}$ remains unchanged.

In this scenario, the DT cone can be replaced by a DT cylinder with increasing density in the axial direction from the point of ignition, with the thermonuclear burn propagating into DT of increasing density.

If the beam stopping by the collective two-stream instability should turn out to be less efficient than predicted by the linear theory, one could change the polarity of the nested double transmission line, generating an intense ion beam instead, with the cathode magnetically insulated against electron emission by the large magnetic field having its source in the current of the outer transmission line.

[7] O. Buneman, Phys. Rev. 115, 503 (1959).

[8] L. Davis, R. Lüst, and A. Schlüter, Z. Naturforsch. 13a, 916 (1958).

[9] F. Winterberg, Atomkernenergie-Kerntechnik 39, 181, 265 (1981).

[10] F. Winterberg, Z. Naturforsch. 54a, 459 (1999). 